\title{
DOUBLE TIME-SCALE IMAGE RECONSTRUCTION OF THE BEATING AND DEVELOPING EMBRYONIC ZEBRAFISH HEART
}

\author{
Michael Liebling ${ }^{1,2}$ Julien Vermot ${ }^{2}$ Scott E. Fraser ${ }^{2}$ \\ ${ }^{1}$ Department of Electrical \& Computer Engineering, University of California, Santa Barbara \\ ${ }^{2}$ Biological Imaging Center, Beckman Institute, California Institute of Technology, Pasadena
}

\begin{abstract}
We present a time-lapse collection and reconstruction technique that allows following embryonic heart development at any computationally halted heart contraction state. The central idea is to image at least one full heartbeat at a fast frame rate, resulting in a two-dimensional plus time $(2 \mathrm{D}+\mathrm{T})$ data set, and repeat this operation every few minutes over several hours for multiple axial positions. The acquired data are five dimensional ( $X, Y$, and $Z$ in space, 'fast' and 'slow' dimensions in time). The (2D+T) image series are then synchronized to their neighbors in the axial and development time dimensions using a non-rigid registration algorithm (constrained such as to leave all but the fast time dimension unchanged). The algorithm proceeds recursively over the different axial positions and developmental stages. We successfully applied this procedure to image the development of the embryonic zebrafish heart between 32 and 44 hours post fertilization (hpf).
\end{abstract}

\section{INTRODUCTION}

Cell motion can occur at various speeds in the developing embryo: relatively slowly, such as for cell migration, division, and growth or much faster, such as for red blood cells moving with the blood flow and heart-wall motion during heart contraction. Velocities can be as slow as a few microns per minute or as fast as several millimeters per second.

In the developing heart, both types of motions are superimposed: the heart is beating while it develops. In the zebrafish, the heart is functional as early as $26 \mathrm{hpf}$, when the heart tube starts pumping blood (about two beats per second [1]), and continues beating while it loops, forms an atrium, a ventricle, valves and eventually reaches maturity after about 5 days post fertilization. This double time scale situation is depicted in Figure 1, where the embryonic heart is schematically represented at different developmental stages and contraction states.

In order to study heart development, acquiring time-lapses in a traditional way-that is, by rapidly acquiring images at

Corresponding author is M. Liebling, Electrical \& Computer Engineering, Mail Code 9560, University of California, Santa Barbara, CA 93106, USA. Email: liebling@ece.ucsb.edu.

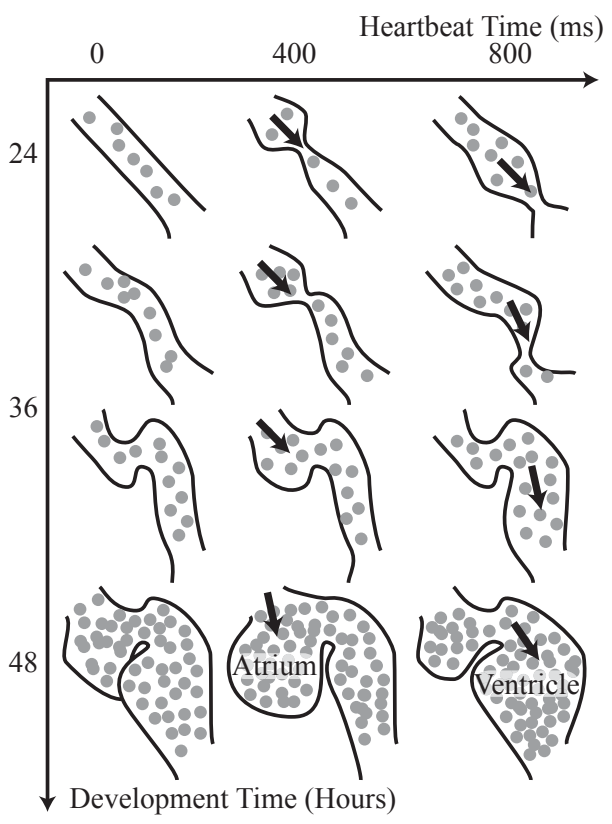

Fig. 1. Since the heart is beating long before it is fully mature and blood flow is known to contribute to its normal development, a double time-scale is necessary to better characterize how the heart forms. Growth and tissue reorganization can be followed along the vertical, slow development time axis at a fixed time in the heartbeat while the dynamics at any given development stage can be investigated along the horizontal, fast heartbeat time axis. Arrows indicate blood flow and gray discs red blood cells.

successive depths and repeating this procedure every few minutes over several hours [see Fig. 2(a)] - is not adequate for the following reasons. First, even the fastest currently available microscopes (for example [2, 3]) are too slow to acquire one full $3 \mathrm{D}$ volume without the heart significantly changing shape between the top and bottom slices. Second, the heart may be in a different contraction state when acquisition resumes every few minutes. Third, imaging a single contraction state at every stage would not allow capturing the dynamics of heart contraction and blood flow, which is essential for reaching a better understanding of how genetic and epige- 
netic factors (such as individual gene defects [4] and patterns of blood flow in the developing heart [5], respectively) interact and contribute to cardiac morphogenesis. The latter point is particularly important for in vivo imaging since the effect of various flow patterns on gene expression and cell morphology has been studied extensively in cultured endothelial cells $[6,7]$ but carrying out similar experiments in vivo has proved to be much more challenging.

We have previously addressed the problem of recording nongated slice-sequences acquired at different axial positions in the living embryonic zebrafish heart and reassembling them to form a dynamic, three-dimensional data volume by temporally synchronizing the sequences, either via uniform [8] or elastic [9] registration. Although we successfully took advantage of this approach to image the heart at several development stages [10], the latter were typically 6-12 hours apart.

Here, we present a technique that allows reducing this time lag to a few minutes. This opens the door to imaging single cardiac cells as they develop on a highly mobile substrate and should allow bridging the gap between genetic and epigenetic studies of cardiac development.

\section{METHOD}

\subsection{Image Acquisition}

We model the measured intensity $I_{m}$ as follows,

$$
\begin{aligned}
I_{m}\left(\mathbf{x}, z_{k}, q_{\ell}, t\right)=\iiint & I\left(\mathbf{x}^{\prime}, z, q_{\ell}+s_{k, l}+t\right) \\
& \times h\left(\mathbf{x}-\mathbf{x}^{\prime}, z_{k}-z\right) \mathrm{d} \mathbf{x}^{\prime} \mathrm{d} z,
\end{aligned}
$$

where $\mathbf{x}=(x, y), z_{k}=k h_{z}, k=0, \ldots, N_{z}$, with $h_{z}$ a fixed axial sampling step and $N_{z}$ the number of slices in the axial direction. Furthermore, we have $q_{\ell}=\ell h_{T_{2}}$, with $\ell=$ $0, \ldots, N_{q}$ and $h_{T_{2}}$ the sampling step in the slow, development time axis. $N_{q}$ is the number of imaged development stages. The optical system's point spread function is denoted $h(\mathbf{x}, z)$. $s_{k, l}$ is an unknown time shift arising from the fact that imaging is started at an arbitrary time-point in the heartbeat cycle. We assume that the four-dimensional intensity function $I(\mathbf{x}, z, t)$, has a period $T$, i.e.,

$$
|I[\mathbf{x}, z, t]-I[\mathbf{x}, z, t+T]| \ll I_{\max } .
$$

For each axial position $z_{k}$ and development stage $q_{\ell}$, we image at least one full heartbeat at a fast frame rate. This procedure is shown in Figure 2(b).

\subsection{Image Reconstruction}

The reconstruction algorithm relies on the fact that over the 'fast' heartbeat time-scale growth or cell migration is negligible and, conversely, that the heartbeat pattern does not significantly change over the few minutes that separate measurements on the 'slow' development time scale. By taking

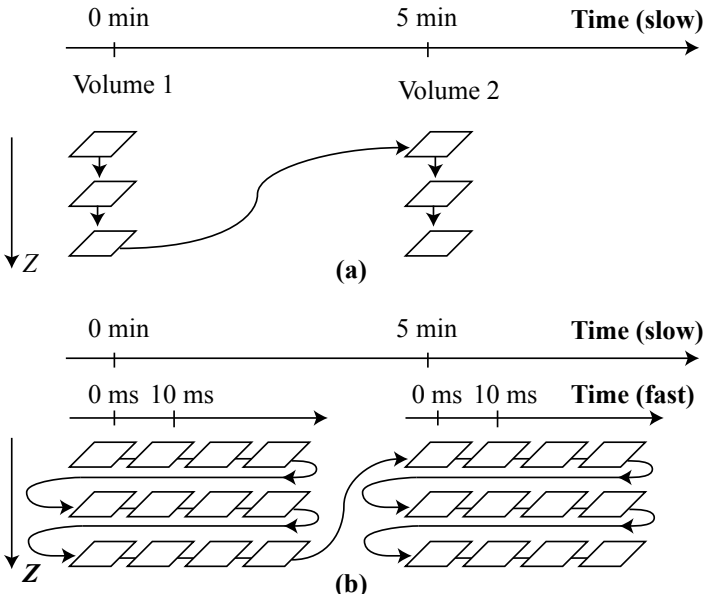

(b)

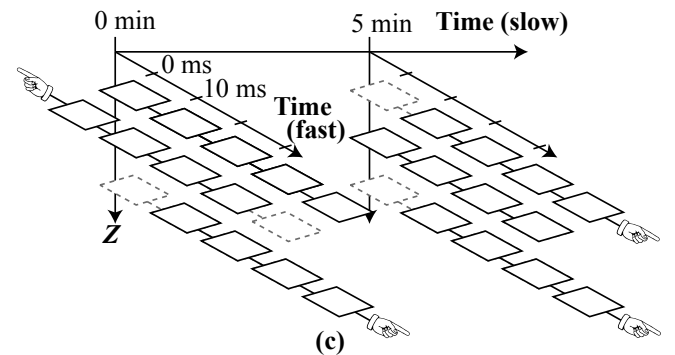

Fig. 2. (a) Traditional time-lapse acquisition of 3D volumes via optical sectioning (e.g. confocal microscopy) is inappropriate for imaging the developing and beating heart since the acquisition of an entire 3D volume cannot be carried out at a sufficient speed. (b) To overcome this limitation, we acquire images at high speed at multiple, but fixed depths, as well as at multiple development time points. (c) Subsequently, the image sequences are synchronized via pixel-based, elastic registration (constrained to the fast time axis) of neighboring (axially or along the slow-time dimension) sequences.

advantage of the repeatedly occurring heart motion, imagesequences from different depths and different development stages can be acquired sequentially and, assuming that the underlying motion is cyclic, reassembled.

In order to recover an estimate $I_{r}\left(\mathbf{x}, z_{k}, q_{\ell}, t\right)$ of $I(\mathbf{x}, z, t)$ from the measurements $I_{m}\left(\mathbf{x}, z_{k}, q_{\ell}, t\right)$, we consider the following objective criterion to measure the discrepancy between the measured data (at depth $z_{k}$ and development time $q_{\ell}$ ) and the synchronized data at neighboring depths $z_{k^{\prime}}$ and development times $q_{\ell^{\prime}}$ (that is, with $k^{\prime}$ in a neighborhood $\mathcal{N}_{k}$ such that $\left|k-k^{\prime}\right|<k_{\text {neigh }}$ (a user-specified value), $\ell^{\prime}$ in a neighborhood $\mathcal{N}_{\ell}$ such that $\ell-\ell^{\prime}<\ell_{\text {neigh }}$ (a user-specified value) and both such that $I_{r}\left(\mathbf{x}, z_{k^{\prime}}, q_{\ell^{\prime}}, t\right)$ has been previously computed):

$$
\begin{aligned}
Q_{k, \ell}(s)=\int_{0}^{L} & \iint_{\mathbf{R}^{2}} \sum_{k^{\prime} \in \mathcal{N}_{k}, \ell^{\prime} \in \mathcal{N}_{\ell}} \mid I_{m}\left(\mathbf{x}, z_{k}, q_{\ell}, t-s\right) \\
& -I_{r}\left(\mathbf{x}, z_{k^{\prime}}, q_{\ell^{\prime}}, t\right) \mid \mathrm{d} \mathbf{x} \mathrm{d} t,
\end{aligned}
$$


where $L$ specifies the length of the time interval over which the alignment is carried out (typically one full heartbeat). We aim at recovering the time shift

$$
\bar{s}_{k, \ell}=\arg \min _{s} Q_{k, \ell}(s)
$$

We start from the depth $z_{\bar{k}}$ and development time $q_{\bar{\ell}}$ for which $\bar{s}_{k, \ell}$ is known (and equal to zero) and find the remaining $\bar{s}_{k, \ell}$ 's recursively.

Similarly to the method presented in [9], we have extended this approach to allow for nonrigid registration, replacing the shifts $s_{k, \ell}$ by warping functions in the objective criterion of Equation (3). Although the registration algorithm described in [9] only takes one (axial) neighbor into account at each step in the recursive registration, the objective criterion (3) is such that the same, dynamic programming algorithm can be used to carry out the minimization here.
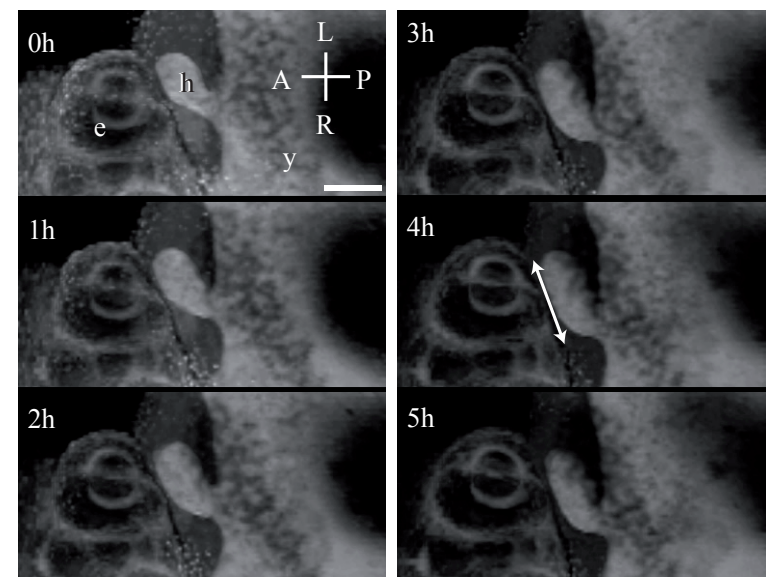

Fig. 3. 5 hour time-lapse of the developing zebrafish heart (between 28 and $33 \mathrm{hpf}$ ), at a fixed point in the heartbeat cycle, after reconstruction with the method described in this paper. The double headed arrows indicates the elongation of the atrium along the left-right axis. Ventral view, maximum intensity projection. e: eye, h: heart, y: yolk sack, L: left, R: right, A: anterior, P: posterior. Scale bar: $100 \mu \mathrm{m}$.

\section{RESULTS}

Wild-type zebrafish (Danio rerio) eggs were spawned using standard techniques [11]. $20 \mathrm{hpf}$ old embryos were soaked in a green fluorescent dye [0.1\% BODIPY FL $\mathrm{C}_{5}$-ceramide (Invitrogen) in artificial pond water] for 8 hours. We acquired two-dimensional image sequences on an inverted laser scanning confocal microscope (LSM 510, Carl Zeiss Jena GmbH, Germany) with a Zeiss Plan-Apochromat 10x/0.45 air microscope objective. We acquired 40 'fast' time points (at 20 frames per second) for $15 Z$ sections ( $8 \mu \mathrm{m}$ apart) and repeated this procedure every 5 minutes for 5 hours. Individual frames had a size of 128 by 64 pixels $(4.8 \mu \mathrm{m}$ lateral sampling size). The images were realigned by use of a MATLAB implementation of the method described above. For a given time point in the heartbeat cycle, the 4D data was then rendered (maximum intensity projection) using commercial visualization software (Imaris, Biplane AG, Zurich, Switzerland).

Six frames from the reconstructed time-lapse are presented in Fig. 3 revealing the elongation of the heart tube. At that stage, the heart elongates along the left-right axis as the atrium increases its size. A detail comparison between the raw, nonsynchronized data and the processed data for four successive development-time images is shown in Fig. 4. Artifacts due to imaging the heart in different contraction states (Fig. 4 (a)) are removed after synchronization (Fig. 4 (b)).

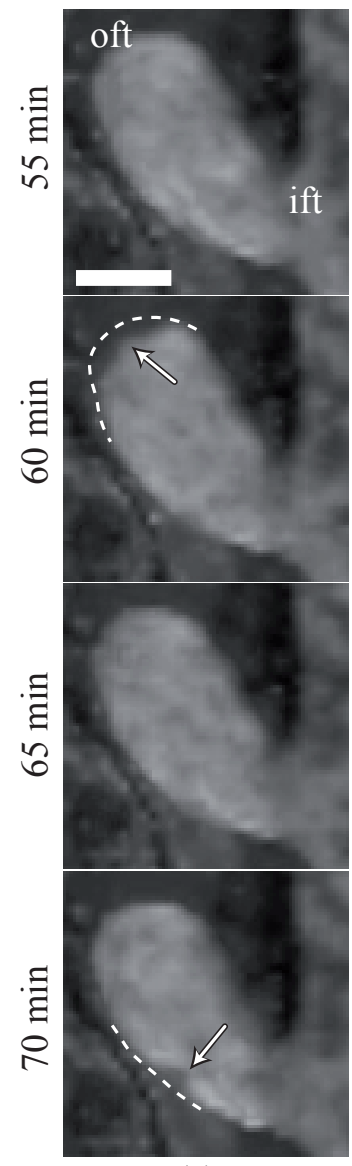

(a)

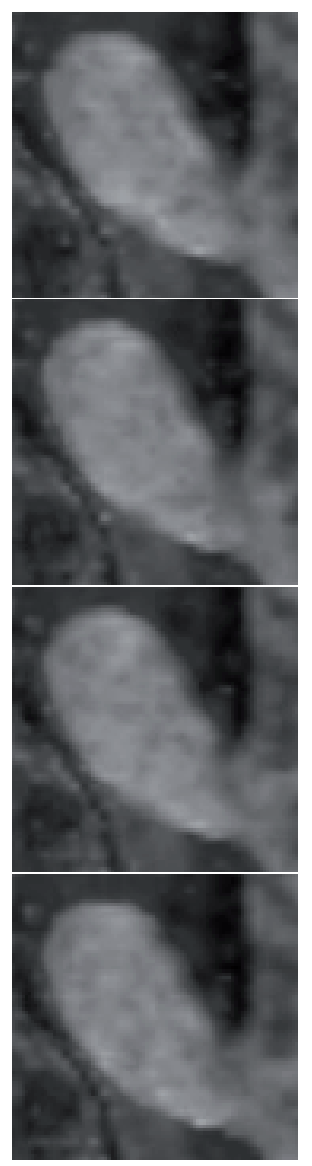

(b)
Fig. 4. (a) Fast heartbeats imply that images acquired at different axial positions and development times are not synchronized. In particular, the heart is in an arbitrary contraction state when acquisition resumes at each (5 minute) development time step (see arrows). (b) After synchronization of the fast sequences along the (heartbeat) time axis, the heart appears to develop in a fixed heartbeat state. This allows for studying changes in heart morphology that are due to growth or cell reorganization rather than heartbeat motion. ift: inflow tract, oft: outflow tract. Scale bar: $50 \mu \mathrm{m}$. 


\section{CONCLUSION}

The presented acquisition and synchronization procedure allows reconstructing volumes of the developing zebrafish heart and following developmental processes without interference from the heartbeat itself. Since it allows imaging the dynamics at a fast time-scale it opens up the possibility of studying the influence of blood flow on heart development simultaneously to gene expression (reported, for example, by a fluorescent protein expressed under the promoter of a gene of interest in a transgenic fish). This might be useful, in particular, for reaching a better understanding of congenital heart disease, the leading cause of infant morbidity in the Western world [4], which often arises during early stages of cardiac development and whose genetic and epigenetic causes remain poorly understood.

Our technique should also be appropriate for imaging heart development with other imaging modalities including nonoptical and macroscopic scales. Further work will include imaging over longer periods of time as well as allowing for the inclusion of temporal landmarks to further constrain the registration.

\section{ACKNOWLEDGMENTS}

M.L. received support from the Swiss National Science Foundation (Fellowship PA002-111433) and J.V. from the Human Frontier Science Program (HFSP). Additional support was provided through NIH/NICHD grant PO1HD037105.

\section{REFERENCES}

[1] A. S. Forouhar, M. Liebling, A. Hickerson, A. NasiraeiMoghaddam, H.-J. Tsai, J. R. Hove, S. E. Fraser, M. E. Dickinson, and M. Gharib, "The embryonic vertebrate heart tube is a dynamic suction pump," Science, vol. 312, no. 5774, pp. 751-753, May 2006.

[2] R. Wolleschensky, B. Zimmermann, and M. Kempe, "High speed confocal fluorescence imaging with a novel line scanning microscope," J. Biomed. Opt., vol. 11, no. 6, pp. 064011 (1-14), Nov.-Dec. 2006.

[3] J. Bewersdorf, R. Pick, and S. W. Hell, "Multifocal mul- tiphoton microscopy," Opt. Lett., vol. 23, pp. 655-657, 1998.

[4] B. G. Bruneau, "The developmental genetics of congenital heart disease," Nature, vol. 451, pp. 943-948, 2008.

[5] J. R Hove, R. W. Köster, A. S. Forouhar, G. AcevedoBolton, S. E. Fraser, and M. Gharib, "Intracardiac fluid forces are an essential epigenetic factor for embryonic cardiogenesis," Nature, vol. 421, no. 6919, pp. 172 177, Jan. 2003.

[6] G. García-Cardeña, J. Comander, K. R. Anderson, B. R. Blackman, and M. A. Gimbrone, Jr., "Biomechanical activation of vascular endothelium as a determinant of its functional phenotype," Proc. Natl. Acad. Sci. USA, vol. 98 , no. 8, pp. 4478-85, 2001.

[7] S. M. McCormick, S. G. Eskin, L. V. McIntire, C. L. Teng, C.-M. Lu, C. G. Russell, and K. K. Chittur, "DNA microarray reveals changes in gene expression of shear stressed human umbilical vein endothelial cells," Proc. Nat. Acad. Sci. USA, vol. 98, no. 16, pp. 8955-8960, 2001.

[8] M. Liebling, A. S. Forouhar, M. Gharib, S. E. Fraser, and M. E. Dickinson, "Four-dimensional cardiac imaging in living embryos via postacquisition synchronization of nongated slice sequences," J. Biomed. Opt., vol. 10, no. 5, pp. 054001 (1-10), Sep./Oct. 2005.

[9] M. Liebling, J. Vermot, A. S. Forouhar, M. Gharib, M. E. Dickinson, and S. E. Fraser, "Nonuniform temporal alignment of slice sequences for four-dimensional imaging of cyclically deforming embryonic structures," in Proceedings of the 3rd IEEE International Symposium on Biomedical Imaging: Macro to Nano (ISBI'06), Arlington, VA, USA, Apr. 6-9, 2006, pp. 1156-1159.

[10] M. Liebling, A. S. Forouhar, R. Wolleschensky, B. Zimmerman, R. Ankerhold, S. E. Fraser, M. Gharib, and M. E. Dickinson, "Rapid three-dimensional imaging and analysis of the beating embryonic heart reveals functional changes during development," Dev. Dynam., vol. 235, no. 11, pp. 2940-2948, Nov. 2006.

[11] M. Westerfield, The Zebrafish Book, University of Oregon Press, Eugene, 1995. 